\title{
Parental Reflectiveness, Posttraumatic Symptoms and Alcohol Use Disorder among Israeli Combat-Veteran Fathers
}

\author{
Daniel Feingold $\mathbb{D}^{1} \cdot$ Gadi Zerach ${ }^{1}$
}

Accepted: 16 June 2021 / Published online: 2 July 2021

(C) The Author(s), under exclusive licence to Springer Science+Business Media, LLC, part of Springer Nature 2021

\begin{abstract}
Combat veterans are highly prone to develop Alcohol Use Disorder (AUD) following their release from duty, presumably due to high prevalence of prolonged aversive emotional symptoms such as Posttraumatic Stress Symptoms (PTSS). Parental Reflective Functions (PRF) and Parental Sense of Competence (PSOC) have been identified as key protective factors in predicting maternal functioning and well-being, yet little is known of its role among fathers, let alone combat veteran fathers. In this study we explored whether PRF and PSOC moderated the association between PTSS and AUD among 189 Israel Defense Forces (IDF) male combat veterans. Participants filled out validated measures assessing PTSS, PRF, PSOC and AUD. Results indicated that PTSS, as well as PRF's "interest and curiosity regarding the child's mental states" subscale, were positively correlated to AUD. In addition, PRF's “certainty about child mental states" subscale moderated the association between PTSS and AUD, so that PTSS and AUD were significantly correlated for participants who reported average or high levels of certainty about their child's mental states. This finding may imply that intrusive mentalizing ("hypermentalizing") by veteran fathers may facilitate the association between PTSS and AUD, presumably by constituting a maladaptive mechanism for coping with the stressful uncertainty embedded in the parent-child relationship.
\end{abstract}

Keywords Combat veterans $\cdot$ Posttraumatic stress symptoms $\cdot$ Alcohol use disorder $\cdot$ Parental reflective functions $\cdot$ Parental sense of competence

\section{Highlights}

- Among Israeli combat-veteran fathers, posttraumatic symptoms were associated with alcohol use disorder.

- Parental reflective functions moderated this association.

- Significate association was observed in participants with average or high levels of certainty about their child's mental states.

\section{Introduction}

Among American military veterans, approximately 56\% report using alcohol monthly (Wagner et al., 2007). Compared to the general population, combat veterans are at significantly greater odds for episodic alcohol use (defined as $\geq 5$ drinks in a two hour period for men) and heavy alcohol use (five or more drinks on the same occasion, on five or more separate occasions; Hoggatt et al., 2017;

\footnotetext{
Daniel Feingold

d.y.feingold@gmail.com

1 Department of Psychology, Ariel University, Ariel, Israel
}

Wagner et al., 2007). Alcohol Use Disorder (AUD) is the most common form of Substance Use Disorder (SUD) among veterans, following tobacco use disorder (Lan et al., 2016), with lifetime and past-year prevalence rates of DSM5 AUD recently estimated at $35.8 \%$ and $15.1 \%$ within this population, respectively (Boden \& Hoggatt, 2018).

It has been suggested that combat veterans are at specifically higher risk for AUD onset compared to nondeployed military personnel (for a review of the literature, see Kelsall et al., 2015), suggesting that combat experience may play a role in veterans' susceptibility to postdeployment alcohol consumption and AUD (Skipper et al., 2014; Wright et al., 2012). Research has suggested that the life threatening and morally ambiguous nature of combat-related experiences may trigger the onset of 
prolonged aversive emotional symptoms, such as Posttraumatic Stress Symptoms (PTSS) and moral injury, which in turn may increase ex-combatants' alcohol use and AUD (Feingold et al., 2019; McDevitt-Murphy et al., 2010; Seal et al., 2011).

Throughout the years, effort has been made to identify factors which may serve as risk/protective factors in the well-established association between PTSS and AUD. In this study, we focused on the potential protective function of parental factor in the PTSS-AUD association. Revisiting Belsky's classic model of parenting, Taraban \& Shaw (2018) highlight the need to take in account fathers' role in the parent-child interaction. Parental factors have been explored historically in the context of mother-child relationships, yet there is a growing body of research addressing fathers' role in parental functioning. For example, it has been suggested that the interest expressed by fathers in their child's internal experience and his/her perspective correlates with higher parental functioning (for example, see Pazzagli, et al., 2018). In a single study focusing on substance use among fathers, high reflective abilities by the parent were negatively correlated with drug-use frequency (Stover \& Kiselica, 2014).

In addition to focusing on fathers, Taraban \& Shaw's revised model of the parenting process suggested that parents' cognitive-affective processes have a significant impact on parenting practices, among mothers and fathers alike. This newly emerging line of evidence indicate that mother's sense of efficacy, as well as their cognitive-affective encoding of their child's behavior and mental states, may play a role the parent process (Lorber et al., 2003; Teti \& Candelaria, 2002). In our we focused on two cognitiveaffective aspects of parenting: parental sense of competence (PSOC), generally defined as parents' trust in their ability to deal with their child (Deković et al., 2010), and parental reflective functioning (PRF), commonly defined as the level of attunement towards one's child, one's parenthood and the parent-child relationship (Håkansson et al., 2019). Naturally, the majority of studies focusing on parental factors explored their protective effect on the child rather than the parent, as a means of fostering responsive and adaptive caregiving and enabling secure attachment (Pazzagli et al., 2018; Rutherford et al., 2017). However, evidence has emerged concerning their role as protective factors for the parents, predominantly by fostering parental satisfaction and well-being (Jones \& Prinz, 2005; Rostad \& Whitaker, 2016). For example, mothers with SUD who exhibited high levels of PRF were less prone to polysubstance abuse and reported later onset of substance use and SUD compared to those with low levels of PRF (Håkansson et al., 2019).

In conclusion, despite the growing interest in fathers' parental functioning, little is known concerning the association between PSOC, PRF and SUDs, as well as the role of PSOC and PRF in moderating the association between PTSS and AUD. This may be important among combat veterans due to the fact that they tend to report feeling less satisfied and competent as parents, more stressed in their parenthood, and more concerned about the future of their children (Christie et al., 2019; Creech \& Misca, 2017).

In Israel, rates of alcohol use and misuse are on the rise (Korn \& Bonny-Noach, 2018; Lev-Ran et al., 2014). Military service in Israel is mandatory, lasting for 36 months for combatants. Israeli combatants are commonly enrolled in conventional warfare duties surrounding the country's borders, as well as routine policing and confrontation with the Palestinian civilian population in the West Bank and Gaza (Yehuda et al., 2015; Zerach \& LeviBelz, 2018). Reported frequency of past-year and pastmonth alcohol use among Israeli combatant veterans is substantially higher compared to the general male population (Feingold et al., 2019). Among Israeli ex-combatants, levels of PTSS have been reported to correlate with frequency of alcohol use, as well as AUD (Yehuda et al., 2015). Therefore, exploring possible factors contributing to the PTSS-AUD association among Israeli veterans is important.

In this study we explored the roles of two parenting domains - PRF and PSOC - among a sample of Israeli male combat veterans, focusing on parental factors' moderating effect on the association between PTSS and AUD. We hypothesized that: (a) PTSS will be positively associated with level of AUD; (b) this association will be moderated by levels of PRF, so that individuals with high levels of reflectiveness will exhibit a weaker association between PTSD and AUD; (c) PSOC will moderate this association, so that individuals with high levels of PSOC will exhibit a weaker association between PTSD and AUD; (d) PTSS will be negatively associated with PRF and PSOC; and (e) PRF and PSOC will be negatively associated with AUD.

\section{Method}

\section{Participants}

Participants were 189 Israel Defense Forces (IDF) male combat veterans. Israeli combatants in the IDF serve a mandatory military service of three years from the age of 18 to 21, with an additional one year or more for officers. Most veterans also remain in reserve combat troops, and are called annually for a period of two weeks to a month to carry out various military missions. Inclusion criteria for this study were a minimum age of 20 years, who served in combat troops and were released from military service within the previous 20 years, and being a parent of at least 
Table 1 Veterans' socio-demographic characteristics

\begin{tabular}{llrr}
\hline Variable & & $N(\%)$ & $M(S D)$ \\
\hline Age at study & & & $30.03(4.16)$ \\
Years of education & & $13.95(2.07)$ \\
Length of marriage & & $7.29(4.46)$ \\
Number of children & & $1.86(0.92)$ \\
Place of birth & Israel & $105(90.5 \%)$ & \\
& Asia/Africa & $2(1.7 \%)$ & \\
& Europe/America & $9(7.7 \%)$ & \\
Marital status & Single & $2(1.8 \%)$ & \\
& Married & $103(90.4 \%)$ & \\
& Divorced & $6(5.3 \%)$ & \\
& Other & $3(2.6 \%)$ & \\
Working status & Full-time job & $71(62.3 \%)$ & \\
& Part-time job & $32(28.1 \%)$ & \\
& Not working & $11(9.6 \%)$ & \\
Income & Above average & $43(38.1 \%)$ & \\
& Average & $19(16.7 \%)$ & \\
& Below average & $52(45.6 \%)$ & \\
Religiosity & Traditional & $19(16.7 \%)$ & \\
& Secular & $33(28.9 \%)$ & \\
& Religious & $60(52.6 \%)$ & \\
& Other & $2(1.8 \%)$ & \\
\hline
\end{tabular}

${ }^{\text {a In years }}$

baverage monthly income was defined as 9200 NIS

one child. Of the 253 participants who gave their consent, 48 (18.9\%) of the participants did not complete study questionnaires, seven (2.7\%) participants were removed due to a fixed response set, and nine (3.5\%) participants did not meet inclusion criteria. In sum, 189 (74.7\%) veteran-fathers comprised the study sample (veterans' socio-demographic characteristics are presented in Table 1).

\section{Procedure}

Potential participants were recruited between January and May 2020 from several settings: volunteers who were active participants in non-formal combat veterans' websites and communities (designated online forums in which veterans can discuss issues relating to combat experiences), students who participated in the study as partial fulfillment of a research participation requirement, and volunteers who responded positively to an advertisement for enrollment in the study. The investigators' research assistants posted a message briefly explaining that they were conducting a research project focusing on military service experiences and asked for possible volunteers. Individuals who agreed to participate received an explanation of the study's aims and a link to the related online survey through an online data-gathering website. Participants were advised that their active participation in the study by completing the questionnaire was an indication of providing their informed consent. Following completion of the survey, participants were sent a letter of thanks and were compensated with a voucher for coffee and pastry (approximate value of $\$ 5$ [USD]). Approval for this study was granted by the "[Masked] University" internal review boards.

\section{Measures}

Posttraumatic stress symptoms. Participants' PTSS were assessed using the Posttraumatic Stress Disorder Checklist (PCL-5; Weathers et al., 2013), which includes the 20 PTSD symptoms listed in the fifth edition of the Diagnostic and Statistical Manual of Mental Disorders (5th ed.; DSM-5; American Psychiatric Association, 2013). Participants were asked to rate how often they suffered from each symptom in the previous month on a five-point scale ranging from 0 (not at all) to 4 (extremely). Specifically, participants were asked about their reactions to any very stressful experiences during their military service (e.g., "I have recurrent dreams and nightmares about a stressful experience from my service"). We operationalized PTSS both as a continuous variable comprised of a total symptom severity score (range: $0-80$ ) by summing the scores for each of the 20 items. Participants were also identified as having PTSS if they exceeded the PCL-5 cut-off point of 33 (Wortmann et al., 2016). Preliminary results show impressive psychometric properties for the PCL-5. For example, its convergent validity was proven when the PCL5 was positively associated with other PTSD measures such as the PDS or DAPS (Blevins, Weathers, Davis, Witte, \& Domino, 2015). The PCL-5 reliability for veterans was Cronbach's $\alpha=0.95$.

Alcohol use disorder. The Alcohol Use Disorders Identification Test (AUDIT; Saunders et al., 1993) is a 10-item measure designed to identify individuals at risk for alcohol use disorder. The measure contains three subscales assessing alcohol consumption (items 1-3(, drinking behaviors (items 4-6), and alcohol-related problems (items 7-10), over the last 12 months. Scores were calculated by summing the scores for the 10 AUDIT questions, ranging from 0 to 40 , with higher scores indicating more problematic alcohol consumption. The AUDIT demonstrates high internal consistency, factorial convergence and criterion validity (DeMartini \& Carey, 2012; Tomás et al., 2017). The Cronbach's alpha value for the AUDIT items was 0.88 .

Parental Reflective Functioning Questionnaire (PRFQ; Luyten et al., 2017). The PRFQ consists of 18 items that participants were asked to rate about their child. The scale is divided into three subscales: (1) Pre-mentalizing modes (PRF-PM) reflects parents' difficulties to understand and interpret the child's mental experience and internal world 
(e.g., "When my child is fussy he or she does that just to annoy me"); (2) Certainty about mental states (PRF-CM) reflects parent's struggle against the opacity of mental states (e.g., "I always know why my child acts the way he or she does"); and (3) Interest and curiosity in mental states (PRFIN). Each statement is rated using a seven-point Likert-type scale from " $1=$ strongly disagree" to " $7=$ strongly agree". The questionnaire showed good psychometric properties in terms of internal consistency (Rutherford et al., 2013) and factor structure (Luyten et al., 2017). Internal consistency (Cronbach's alpha) was $0.88,0.78$, and 0.71 for the prementalizing, certainty, and interest and curiosity subscales, respectively.

Parenting Sense of Competence Scale (PSOC; GibaudWallston \& Wandersman, 1978; Johnston \& Mash, 1989). The PSOC assess parents' confidence in their ability to parent. The scale consists of 16 items tapping parents' satisfaction, interest and efficacy in their parental role. Each statement is rated using a six-point Likert-type scale ranging from " $1=$ strongly disagree" to " $6=$ strongly agree". Total scores range from 16 to 96 , with a higher sum score indicating greater sense of competence in parenting. The PSOC showed good psychometric properties in terms of internal consistency, test-retest reliability, and convergent validity with measures of parental distress among veterans' population (Bui et al., 2017).

Combat experiences. Combat experiences were examined by the 18-item Combat Experiences Scale (CES; Hoge et al., 2004), which comprises conventional modern combat-related experiences to which an individual may have been exposed (e.g., being attacked or ambushed, shooting or directing fire at the enemy, handling or uncovering dead bodies or body parts). Respondents were asked to indicate which events they had experienced at any time during a deployment; this resulted in a total number of combat experiences, ranging from 0 to 18 . In the current sample, the Cronbach's alpha value for the CES items was 0.85 .

Socio-demographics were collected from all participants for the following variables: age, country of birth, education, religiosity, income, length of relationship with partner, and number of children.

\section{Data Analysis}

Data analysis was divided into five stages. First, descriptive statistics and rates of PTSS, and AUD were calculated. Second, the associations between the study variables were examined with a series of Pearson correlation analyses. Third, the data were screened for missing values. The percentage of missing values in the studied variables ranged from $15.3 \%$ to $36 \%$. The data was missing completely at random (MCAR), Little's $\chi^{2}(8),=3.71, p=0.88$. Missing data were handled with the Expectation-Maximization (EM) module of IBM SPSS software (Version 23). Fourth, in order to address the unique contribution of the independent variables to AUD, a four-step hierarchical regression analysis was conducted.

All independent variables were centered before entering into regression models. In the first step of the regression, we entered the sociodemographic variables of length of relationship for statistical control. In the second step, we entered the sum score of combat exposure and the psychopathological correlate of AUD (PTSS). In the third step, we entered the PRF dimensions of parental pre-mentalizing (PM), certainty (CM), and interest (IN). In the fourth step, we entered the hypothesized two-way interactions between PTSS and parental reflective functioning dimensions. Finally, in order to specifically examine our moderation hypotheses, ordinary least squares regression analysis was conducted using the PROCESS macro in SPSS (Hayes, 2012). We tested the significance of interaction effects with a "pick-a-point" approach for probing moderation effects, with standardized scores for the study variables. This approach involves selecting representative moderator values (e.g., low $=$ one standard deviation below the mean, moderate $=$ sample mean, and high $=$ one standard deviation above the mean), and then estimating the effect of the focal predictor at those values (Hayes \& Matthes, 2009).

\section{Results}

\section{Prevalence of PTSS and AUD}

Regarding the PCL-C-5, 14 participants (8.9\%) exceeded the recommended cutoff score of 33 for a diagnosis of PTSD. There were 20 participants $(12.5 \%)$ who reported fully symptomatic self-reported probable PTSD following stressful experiences in military service, according to the DSM-5 diagnostic criteria (APA, 2013). The self-report diagnosis of problematic alcohol use, as defined by the AUDIT-10 suggested cutoff, was set to equal or lower than a sum score of 7 (low-risk), 8-15 (risky or hazardous level), 16-19 (high-risk or harmful level), and higher than 20 (high risk with high likelihood of dependence) (Saunders et al., 1993). Accordingly, the prevalence of low-risk in our sample was $88.7 \%(n=134)$, risky or hazardous level prevalence was $8.6 \%(n=13)$; high-risk or harmful level prevalence was $0.7 \%(n=1)$; and high risk with high likelihood of dependence prevalence was $2 \%(n=3)$.

\section{Associations between the Study Variables}

As seen in Table 2, the results partially confirmed our hypothesis. There was a positive association between PTSS 
Table 2 Descriptive statistics and bivariate correlations between the study variables

\begin{tabular}{lllllllc}
\hline & 1 & 2 & 3 & 4 & 5 & 6 & 7 \\
\hline 1. Combat exposure & - & & & & & \\
2. PTSS & $0.39^{* * *}$ & - & & & & \\
3. PRFQ-Pre-mentalizing & $0.22^{* *}$ & $0.46^{* * *}$ & - & & & \\
4. PRFQ-Certainty & $0.28^{* *}$ & 0.11 & 0.08 & - & & \\
5. PRFQ-Interest & 0.13 & -0.11 & 0.07 & $0.38^{* * *}$ & - & & \\
6. PSOC & 0.03 & $-0.38^{* * *}$ & $-0.53^{* * *}$ & $0.31^{* * *}$ & $0.47^{* * *}$ & - & \\
7. Alcohol misuse & 0.10 & $0.48^{* * *}$ & $0.29 * *$ & -0.22 & $-0.37^{* * *}$ & $-0.36^{* * *}$ & - \\
$M$ & 5.10 & 13.40 & 2.41 & 3.79 & 5.13 & 73.42 & 3.63 \\
$S D$ & 4.06 & 13.76 & 0.93 & 0.85 & 1.00 & 10.00 & 4.18 \\
\hline
\end{tabular}

$P R F Q$ parental reflective functioning questionnaire, $P S O C$ parenting sense of competence scale

$* * p<0.01 ; * * * p<0.001$ and total sum of alcohol misuse. Moreover, PTSS was positively associated with PRF-PM, but not with PRF-CM and PRF-IN. PTSS was also negatively associated with lower levels of parental sense of competence. Importantly, alcohol misuse was positively associated with parental PM and negatively associated with PRF-IN and PSOC, but not with PRF-CM. We also note that PSOC was negatively associated with PRF-PM and positively associated with PRF-CM and PRF-IN. Preliminary analyses performed before we examined the hypotheses, showed that alcohol misuse among veterans was not correlated with any of our demographic variables, including age, family status, income level, religiosity, or education level. However, we did find that length of marital relationship was negatively correlated with PTSS $(r=-0.24, p<0.00)$. Thus, this variable has been entered into the regression models for statistical control.

\section{Prediction of AUD by Combat Exposure, PTSS, Parental Competence and Parental Reflective Functioning}

Our next aims were to examine the relative contributions of combat exposure, PTSS, PRF and PSOC to AUD. Furthermore, we examined the moderating role of parental reflective functioning dimensions and parental sense of competence on the associations between participants' PTSS and AUD.

The total set of variables in the final model explained $40.3 \%$ of the variance of participants' AUD. As seen in Table 3, in the final model we found that participants' PTSS positively contributed to higher levels of AUD. Furthermore, the PRF-IN dimension was a significant negative predictor of AUD, beyond the contribution of combat exposure and PTSS. Thus, the higher levels that veterans reported of reflective parenting as manifested in parental interest and curiosity in children's mental status, the less likely they were to endorse AUD. Finally, as hypothesized, we found a two-way significant interaction between PTSS and PRF-CM.

Following the hierarchical regression results, we employed moderation analysis using the PROCESS macro (Hayes, 2012). PTSS was the independent variable and parental reflective functioning dimensions were entered separately as moderating variables. This analysis helped us understand the specific role of each parenting dimensions in the association between PTSS and AUD, and to specify the ways parental reflective functioning dimensions contribute to AUD following exposure to combat. All other variables, including the other reflective functioning dimensions, were entered as covariates. As reported in Table 3, a significant interaction was found between PTSS and PRF-CM, $b=$ 0.22 , CI [0.07, 0.37], $\mathrm{SE}=0.08, \mathrm{t}(1181)=2.89, p=0.004$, $95 \%$. Probing of the interaction revealed that significant positive effects were revealed for PTSS on their AUD for average PRF-CM, $b=0.41$, CI $[0.26,55] \mathrm{SE}=0.07, \mathrm{t}$ $(181)=5.52, p=0.001,95 \%$; and for high PRF-CM, $b=$ 0.59 , CI [0.42, 0.75], $\mathrm{SE}=0.08, \mathrm{t}(181)=6.94, p=0.001$, 95\%; but not for low PRF-CM, $b=0.17$, CI [ $-0.01,0.36$, $\mathrm{SE}=0.09, \mathrm{t}(181)=1.87, p=0.07,95 \%$; (see Fig. 1).

\section{Discussion}

In this study we sought to explore the moderating role of PSOC and PRF on the association between PTSS and AUD among Israeli combat veteran fathers. Our main findings indicated that PTSS were positively and significantly associated with AUD and that PRF-interest and curiosity regarding children's mental states was negatively and significantly associated with AUD. In addition, PRF-certainty about children's mental states moderated the association between PTSS and AUD, so that PTSS and AUD were not significantly correlated only for participants who reported low certainty about their children's mental states. To the best of our knowledge, this study is the first to indicate 
Table 3 Summary of hierarchical regression coefficients of current alcohol misuse with length of relations, combat exposure, posttraumatic stress symptoms (PTSS), parental reflective functioning and parenting sense of competence.

\begin{tabular}{|c|c|c|c|c|c|c|c|c|c|c|c|c|}
\hline \multicolumn{13}{|l|}{ Alcohol Misuse } \\
\hline & \multicolumn{3}{|c|}{ Model $1^{\mathrm{a}}$} & \multicolumn{3}{|c|}{ Model $2^{b}$} & \multicolumn{3}{|c|}{ Model $3^{\mathrm{c}}$} & \multicolumn{3}{|c|}{ Model $4^{\mathrm{d}}$} \\
\hline & $b$ & $S E b$ & $\beta$ & $b$ & $S E b$ & $\beta$ & $b$ & $S E b$ & $\beta$ & $b$ & $S E b$ & $\beta$ \\
\hline Length of relationship & -0.16 & 0.07 & -0.16 & -0.02 & 0.07 & -0.02 & -0.02 & 0.07 & -0.02 & -0.01 & 0.07 & -0.01 \\
\hline Combat exposure & & & & -0.10 & 0.07 & -0.10 & -0.06 & 0.07 & -0.06 & -0.06 & 0.07 & -0.06 \\
\hline PTSS & & & & 0.52 & 0.07 & $0.52 * * *$ & 0.41 & 0.07 & $0.41 * * *$ & 0.26 & 0.09 & $0.26 * *$ \\
\hline PRFQ-Pre-mentalizing & & & & & & & 0.05 & 0.08 & 0.05 & -0.09 & 0.08 & -0.09 \\
\hline PRFQ-Certainty & & & & & & & 0.07 & 0.09 & 0.07 & 0.08 & 0.07 & 0.08 \\
\hline PRFQ-Interest & & & & & & & -0.33 & 0.07 & $-0.33 * * *$ & -0.34 & 0.07 & $-0.34 * * *$ \\
\hline PSOC & & & & & & & -0.05 & 0.09 & -0.05 & -0.10 & 0.08 & -0.10 \\
\hline PTSS x PM & & & & & & & & & & 0.05 & 0.06 & 0.11 \\
\hline PTSS x CM & & & & & & & & & & 0.22 & 0.07 & $0.29 * *$ \\
\hline PTSS x IN & & & & & & & & & & -0.12 & 0.07 & -0.13 \\
\hline PTSS x PSOC & & & & & & & & & & -0.06 & 0.07 & -0.07 \\
\hline
\end{tabular}

$P R F Q$ parental reflective functioning questionnaire, $P S O C$ parenting sense of competence scale, $P M$ pre-mentalizing modes, $C M$ certainty about child mental states, $I N$ interest and curiosity regarding child mental states

$* * p<0.01 ; * * * p<0.001$

${ }^{\mathrm{a}} \Delta R^{2}=0.3 \% ; \Delta F(1187)=4.98 *$

${ }^{\mathrm{b}} \Delta R^{2}=21.6 \% ; \Delta F(2185)=26.42 * * *$

${ }^{\mathrm{c}} \Delta R^{2}=10.7 \% ; \Delta F(1181)=7.41 * * *$

${ }^{\mathrm{d}} \Delta R^{2}=8.9 \% ; \Delta F(1177)=7.01 * * *$

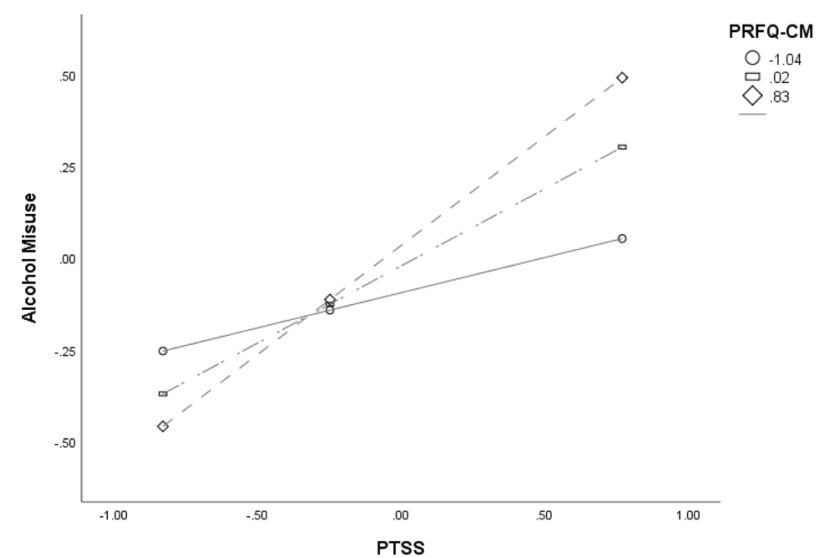

Fig. 1 Moderation of the association between PTSS and Alcohol misuse by parental reflective functioning-certainty about child mental states dimension. Note. PRFQ parental reflective functioning questionnaire, CM certainty about child mental states

possible parenting domains that might attenuate the deleterious impact of veterans fathers' PTSS on problematic alcohol use.

Our findings indicate that PTSS were significantly associated with higher levels of AUD. This is in line with numerous studies which have indicated that PTSS and PTSD are highly correlated with excessive alcohol use and AUD (Pietrzak et al., 2011). This is particularly true for combat veterans, who are highly inclined toward PTSS due to high combat exposure, and are therefore highly prone to AUD (Lan et al., 2016). Several explanations were raised for the high co-occurrence of PTSS and AUD. Alcohol use has often been regarded as a means for "self-medicating" PTSS, particularly intrusive memories, avoidance and sleep disturbances (Hawn et al., 2020). It is now thought that selfmedication of PTSS with alcohol may paradoxically lead to a gradual transition to AUD, as negative physical and emotional symptoms associated with alcohol withdrawal may lead to a compulsive pattern of alcohol use (Koob et al., 2020). In this line, it has been reported recently that experiential avoidance, i.e., the tendency to avoid experiencing negative affective states (Chawla \& Ostafin, 2007), moderated the association between PTSS and AUD among Israeli combat veterans. According to this study, PTSS were significantly correlated with AUD veterans who exhibited high or average, but not low, levels of experiential avoidance (Feingold \& Zerach, 2020).

In our study, higher levels of PTSS were associated with decreased fathers' sense of competence. The association between PTSS and decreased PSOC and sense of parental efficacy were previously reported among U.S. veteran parents (Gewirtz et al., 2010; Sherman et al., 2016), with recent evidence suggesting that PSOC may mediate the association between PTSS and family functioning (Laifer et al., 2019). Notably, there is growing support for the notion that mothers' ability to mentalize on their own trauma, and not 
the severity of their PTSS, predicts the levels of maternal PRF (Allen, 2018; Schechter et al., 2005). Accordingly, no significant correlations were reported between PTSS and PRF's "certainty" and "interest" subscales in our study. However, PTSS did significantly correlate with PRF's prementalizing modes in our study, suggesting that fathers' PTSS are associated with increased difficulties in understanding and interpreting the child's mental experience and internal world. This finding may be attributed to the poorer general mentalizing abilities previously reported among men compared to women (Adenzato et al., 2017; Krach et al., 2009; McClure, 2000).This would suggest that veteran fathers may not acquire sufficient mental representation of their own trauma, thus resulting in PTSS interfering with their parental mentalization ability. However, this requires further exploration.

Notably in our study, certainty about children's mental states moderated the association between PTSS and AUD, so that high levels of certainty were associated with a significant PTSS-AUD correlation. It has been suggested that high certainty about the child's mental states may in fact represent a maladaptive pattern of intrusive mentalizing or hypermentalizing by the parent (Luyten et al., 2017). High levels of certainty about the child's mental states and intrusive mentalizing have previously been documented among mothers with PTSD (Suardi et al., 2020), as well as mothers and fathers with Attention Deficit and Hyperactive Disorder (ADHD; Mazzeschi et al., 2019). This suggests that high levels of mental distress may be associated with a tendency to hypermentalize on the child, presumably as a compensatory mechanism for the innate uncertainty embedded in the parent-child relationship (Mazzeschi et al., 2019). Therefore, it may well be that in our study, PTSS of intrusion and hyperarousal may have enhanced participants' distress concerning their child's mental states, resulting in increased effort to self-regulate this distress by hypermentalizing concerning the child's mental states. As maladaptive emotional regulation has been identified as a key component associated with AUD in the general population and among veterans (Feingold \& Zerach, 2020; Koob et al., 2020), this effort to self-regulate fathers' ambiguity regarding his child's emotional state might paradoxically lead to increased AUD.

A noteworthy finding in our study was that levels of PRF's 'interest and curiosity regarding the child's mental states' subscale were negatively correlated with AUD after controlling for possible confounders. Maternal interest in her child's mental states was previously associated with greater attention and tolerance to the child's distress (Rutherford et al., 2013; Rutherford et al., 2017), presumably due to the mediating effect of executive functions (Rutherford et al., 2018). Previous studies have indicated that interest in the child's mental states was also positively correlated with parent satisfaction, involvement, concern and communication with the child (Rostad \& Whitaker, 2016). Interest in the child's mental states has shown a crude positive correlation with PSOC in our study. Therefore, it may well be that in our sample levels of interest in the child's mental states were negatively associated with AUD via a possible mediating effect of decreased parental satisfaction, low sense of competence or a poor parent-child relationship. However, this should be further explored in future studies. In addition, due to the cross-sectional nature of this study, an inverse notion should be considered, in which elevated AUD may have affected participants' levels of interest in their child's mental status due to the long-term effect of alcohol use and AUD on cognition, affect and interpersonal relationships (Koob et al., 2020).

Surprisingly, PSOC did not moderate the association between PTSS and AUD in our study. However, PSOC did exhibit significant negative crude associations with PTSS and AUD. Firstly, it is possible that due to inclusion of PSOC and PRF in the same regression decreased the power to detect an interaction between PTSS and PSOC. Secondly, several studies have emphasized the mediating role of PSOC, predominantly as a mechanism of positive change (Deković et al., 2010; Egberts et al., 2015). Among combat veterans, PTSS may lead to decreased PSOC, presumably due to the effect of negative cognitions towards the self and/ or decreased parental resources, which in turn may increase levels of AUD. However, this should be explored further in future research

This study has several limitations which should be acknowledged. First and foremost, our findings are based on non-representative convenience sample of Israeli combat veterans, implying that caution should be used when considering our results. For example, it may well be that combatant veterans participating in online forums are exposed to reflective discourse concerning PTSS and parenthood and those who are enrolled in academic studies possess relatively high general sense of competence and relatively low levels of AUD. Therefore, our findings do not necessarily represent under-competent or highly alcoholdependent veteran populations. Second, as mentioned above, the cross-sectional nature of this study does not allow for establishing directionality or causality of the association between parental factors and AUD. Third, despite the contribution of our study to the emerging research on fatherhood and its specific correlates among combat veterans, gender differences are prominent in AUD (Keyes et al., 2011). Female combatants are being assigned to combat positions in recent years both in the U.S. and the IDF, and are attracting growing academic attention (Finley, 2016; Harel-Shalev \& Daphna-Tekoah, 2015). Therefore, our results could not be generalized to the female veteran population. Furthermore, the validity of instruments used in 
this study is limited. The AUDIT has shown good reliability and validity, yet it cannot attribute a formal diagnosis of AUD, as it lacks indication of a clinically significant impairment or distress caused directly by a maladaptive pattern of substance use, and it is not based on a face to face interview (American Psychiatric Association, 2000). The PRFQ and PSOC, despite being practical, validated and widely used, are based on self-report are therefore highly bias prone compared to alternative measures (Fonagy et al., 1991). Finally, data was collected in part during the first COVID-19 outbreak in Israel ( March 2020). Increase in PTSS levels and alcohol consumption has been reported globally throughout 2020-2021 (Mallet et al., 2020; Xiong et al., 2020), therefore it may well be that levels of these variables were inflated a-priori in our study. However, this was not directly assessed in our study and may have not been present so early during the COVID19 outbreak.

Despite these limitations, and given the scarce research regarding veterans as fathers, the present study is one of the first to explore the role of parental characteristics in this association between PTSS and AUD among combat veterans. As the ex-combatant population is highly prone to psychological and behavioral pathology, much effort has been made to identify protective factors which may facilitate a more adaptive adjustment to civilian life. Israel has the highest fertility rate among developed countries (OECD, 2020), and parenthood is considered an important societal and religious value among men and women alike (Okun, 2013). As such, our results may indicate that promoting adaptive PRF may enhance male veterans' resilience to AUD, presumably by providing alternative mechanisms for coping with the stressful uncertainty which is embedded in the parent-child relationship. Unfortunately, to date there is conflicting evidence concerning the efficacy of interventions designated to enhance PRF, whether by using mentalization-based therapy, early dyadic interventions or group therapy (Barlow et al., 2020; Camoirano, 2017; Lo \& Wong, 2020). Future interventions should be adapted to suit parents who are combat veterans, embraced and assimilated by the Veteran Administration. Specifically, experiencefocused intervention, such as Schema-focused therapy and Somatic Experience, may be beneficial as they can allow patients to relieve and work-through combat-related traumatic memories and its emotional, cognitive and physical imprints, in order to modify current response patterns to daily-life parental experiences and conflicts (Cockram et al., 2010; Payne et al., 2015).

\section{Compliance with Ethical Standards}

Conflict of Interest The authors declare no competing interests.
Consent to Participate Informed consent was obtained from all individual participants included in the study.

Publisher's note Springer Nature remains neutral with regard to jurisdictional claims in published maps and institutional affiliations.

\section{References}

Adenzato, M., Brambilla, M., Manenti, R., De Lucia, L., Trojano, L., Garofalo, S., Enrici, I., \& Cotelli, M. (2017). Gender differences in cognitive Theory of Mind revealed by transcranial direct current stimulation on medial prefrontal cortex. Scientific Reports, 7 (1), 1-9.

Allen, J. G. (2018). Mentalizing in the development and treatment of attachment trauma. Routledge.

American Psychiatric Association. (2000). Diagnostic and statistical manual of mental disorders (4th ed.). Text Revision (DSM-IVTR). American Psychiatric Association.

American Psychiatric Association. (2013). Diagnostic and statistical manual of mental disorders (5th ed.). American Psychiatric Publishing.

Barlow, J., Sleed, M., \& Midgley, N. (2020). Enhancing parental reflective functioning through early dyadic interventions: a systematic review and meta-analysis. Infant Mental Health Journal. https://doi.org/10.1002/imhj.21896.

Blevins, C. A., Weathers, F. W., Davis, M. T., Witte, T. K., \& Domino, J. L. (2015). The posttraumatic stress disorder checklist for DSM-5 (PCL-5): development and initial psychometric evaluation. Journal of Traumatic Stress, 28(6), 489-498.

Boden, M. T., \& Hoggatt, K. J. (2018). Substance use disorders among veterans in a nationally representative sample: prevalence and associated functioning and treatment utilization. J Stud Alcohol Drugs, 79(6), 853-861.

Bui, E., Zakarian, R. J., Laifer, L. M., Sager, J. C., Chen, Y., Cohen, S., ... \& Ohye, B. (2017). Psychometric properties of the Parenting Sense of Competence Scale in treatment-seeking post-9/11 veterans. Journal Child \& Family Studies, 26(2), 464-470.

Camoirano, A. (2017). Mentalizing makes parenting work: a review about parental reflective functioning and clinical interventions to improve it. Frontiers in Psychology, 8, 14.

Chawla, N., \& Ostafin, B. (2007). Experiential avoidance as a functional dimensional approach to psychopathology: an empirical review. Journal of Clinical Psychology, 63(9), 871-890.

Christie, H., Hamilton-Giachritsis, C., Alves-Costa, F., Tomlinson, M., \& Halligan, S. L. (2019). The impact of parental posttraumatic stress disorder on parenting: a systematic review. European journal of psychotraumatology, 10(1), 1550345.

Cockram, D. M., Drummond, P. D., \& Lee, C. W. (2010). Role and treatment of early maladaptive schemas in Vietnam veterans with PTSD. Clinical Psychology \& Psychotherapy: An International Journal of Theory \& Practice, 17(3), 165-182.

Creech, S. K., \& Misca, G. (2017). Parenting with PTSD: a review of research on the influence of PTSD on parent-child functioning in military and veteran families. Frontiers in Psychology, 8, 1101.

Deković, M., Asscher, J. J., Hermanns, J., Reitz, E., Prinzie, P., \& Van Den Akker, A. L. (2010). Tracing changes in families who participated in the home-start parenting program: parental sense of competence as mechanism of change. Prevention Science, 11(3), 263-274.

DeMartini, K. S., \& Carey, K. B. (2012). Optimizing the use of the AUDIT for alcohol screening in college students. Psychological Assessment, 24(4), 954. 
Egberts, M. R., Prinzie, P., Deković, M., de Haan, A. D., \& van den Akker, A. L. (2015). The prospective relationship between child personality and perceived parenting: mediation by parental sense of competence. Personality and Individual Differences, 77, 193-198.

Feingold, D., \& Zerach, G. (2020). Emotion regulation and experiential avoidance moderate the association between posttraumatic symptoms and alcohol use disorder among israeli combat veterans. Addictive Behaviours, 115, 106776.

Feingold, D., Zerach, G., \& Levi-Belz, Y. (2019). The association between moral injury and substance use among israeli combat veterans: the mediating role of distress and perceived social support. International Journal of Mental Health and Addiction, 17(2), 217-233.

Finley, E. P. (2016). The few, the proud: women combat veterans and post-traumatic stress disorder in the United States. Global Mental Health: Anthropological. Perspectives, 2, 221.

Fonagy, P., Steele, M., Steele, H., Moran, G. S., \& Higgitt, A. C. (1991). The capacity for understanding mental states: the reflective self in parent and child and its significance for security of attachment. Infant Mental Health Journal, 12(3), 201-218.

Gewirtz, A. H., Polusny, M. A., DeGarmo, D. S., Khaylis, A., \& Erbes, C. R. (2010). Posttraumatic stress symptoms among National Guard soldiers deployed to Iraq: associations with parenting behaviors and couple adjustment. Journal of Consulting and Clinical Psychology, 78(5), 599.

Gibaud-Wallston, J., \& Wandersman, L. P. (1978). Parenting Sense of Competence (PSOC) scale modified.

Harel-Shalev, A., \& Daphna-Tekoah, S. (2015). Gendering conflict analysis: analysing Israeli female combatants' experiences. Female combatants in conflict and peace (pp. 69-83). Springer.

Hayes, A. F. (2012). PROCESS: A versatile computational tool for observed variable mediation, moderation, and conditional process modeling..

Hawn, S. E., Cusack, S. E., \& Amstadter, A. B. (2020). A systematic review of the self-medication hypothesis in the context of posttraumatic stress disorder and comorbid problematic alcohol use. Journal of Traumatic Stress. https://doi.org/10.1002/jts.22521.

Hayes, A. F., \& Matthes, J. (2009). Computational procedures for probing interactions in OLS and logistic regression: SPSS and SAS implementations. Behavior Research Methods, 41(3), 924-936.

Hoge, C. W., Castro, C. A., Messer, S. C., McGurk, D., Cotting, D. I., \& Koffman, R. L. (2004). Combat duty in Iraq and Afghanistan, mental health problems, and barriers to care. New England Journal of Medicine, 351(1), 13-22.

Hoggatt, K. J., Lehavot, K., Krenek, M., Schweizer, C. A., \& Simpson, T. (2017). Prevalence of substance misuse among US veterans in the general population. The American Journal on Addictions, 26(4), 357-365.

Håkansson, U., Watten, R. G., Söderström, K., \& Øie, M. G. (2019). The association between executive functioning and parental stress and psychological distress is mediated by parental reflective functioning in mothers with substance use disorder. Stress and Health, 35(4), 407-420.

Jones, T. L., \& Prinz, R. J. (2005). Potential roles of parental selfefficacy in parent and child adjustment: a review. Clinical Psychology Review, 25(3), 341-363.

Johnston, C. \& Mash, E. J. (1989). A measure of parenting satisfaction and efficacy. Journal clinical child psychology, 18(2), 167-175.

Kelsall, H. L., Wijesinghe, M. S. D., Creamer, M. C., McKenzie, D. P., Forbes, A. B., Page, M. J., \& Sim, M. R. (2015). Alcohol use and substance use disorders in Gulf War, Afghanistan, and Iraq War veterans compared with nondeployed military personnel. Epidemiologic Reviews, 37(1), 38-54.

Keyes, K. M., Li, G., \& Hasin, D. S. (2011). Birth cohort effects and gender differences in alcohol epidemiology: a review and synthesis. Alcoholism: Clinical and Experimental Research, 35 (12), 2101-2112.

Koob, G. F., Powell, P., \& White, A. (2020). Addiction as a coping response: hyperkatifeia, deaths of despair, and COVID-19. American Journal of Psychiatry, 177(11), 1031-1037.

Korn, L., \& Bonny-Noach, H. (2018). Gender differences in deviance and health risk behaviors among young-adults undergraduate students. Substance Use \& Misuse, 53(1), 59-69.

Kotz, D., Viechtbauer, W., Simpson, C., van Schayck, O. C., West, R., \& Sheikh, A. (2015). Cardiovascular and neuropsychiatric risks of varenicline: a retrospective cohort study. The Lancet Respiratory Medicine, 3(10), 761-768. https://doi.org/10.1016/ S2213-2600(15)00320-3.

Krach, S., Blümel, I., Marjoram, D., Lataster, T., Krabbendam, L., Weber, J., van Os, J., \& Kircher, T. (2009). Are women better mindreaders? Sex differences in neural correlates of mentalizing detected with functional MRI. BMC Neuroscience, 10(1), 9.

Laifer, L. M., Blackburn, A. M., Goetter, E. M., Ohye, B. Y., Simon, N. M., \& Bui, E. (2019). Potential mediating role of parenting competence in the relationship between posttraumatic stress disorder and family functioning post-9/11 veteran parents. Journal of Child and Family Studies, 28(7), 1843-1849.

Lan, C. W., Fiellin, D. A., Barry, D. T., Bryant, K. J., Gordon, A. J., Edelman, E. J., Gaither, J. R., Maisto, S. A., \& Marshall, B. D. (2016). The epidemiology of substance use disorders in US Veterans: a systematic review and analysis of assessment methods. The American Journal on Addictions, 25(1), 7-24.

Lev-Ran, S., Florentin, I., Feingold, D., \& Rehm, J. (2014). Individuals receiving specialized treatment for drug and alcohol dependence and gambling disorder in Israel-characteristics and implications for prevalence estimates. Substance Abuse, 35(3), 268-275. https://doi.org/10.1080/08897077.2014.907860.

Lo, C. K., \& Wong, S. Y. (2020). The effectiveness of parenting programs in regard to improving parental reflective functioning: a meta-analysis. Attachment \& Human Development, 4, 1-17.

Lorber, M. F., O'Leary, S. G., \& Kendziora, K. T. (2003). Mothers' overreactive discipline and their encoding and appraisals of toddler behavior. Journal of Abnormal Child Psychology, 31(5), 485-494.

Luyten, P., Mayes, L. C., Nijssens, L., \& Fonagy, P. (2017). The parental reflective functioning questionnaire: development and preliminary validation. PLOS ONE, 12(5), e0176218.

Mallet, J., Dubertret, C., \& Le Strat, Y. (2020). Addictions in the COVID-19 era: current evidence, future perspectives a comprehensive review. Progress in Neuro-Psychopharmacology and Biological Psychiatry, 106, 110070.

Mazzeschi, C., Buratta, L., Cavallina, C., Ghignoni, R., Margheriti, M., \& Pazzagli, C. (2019). Parental reflective functioning in mothers and fathers of children with ADHD: issues regarding assessment and implications for intervention. Frontiers in Public Health, 7, 263.

McClure, E. B. (2000). A meta-analytic review of sex differences in facial expression processing and their development in infants, children, and adolescents. Psychological Bulletin, 126(3), 424.

McDevitt-Murphy, M. E., Williams, J. L., Bracken, K. L., Fields, J. A., Monahan, C. J., \& Murphy, J. G. (2010). PTSD symptoms, hazardous drinking, and health functioning among US OEF and OIF veterans presenting to primary care. Journal of Traumatic Stress: Official Publication of The International Society for Traumatic Stress Studies, 23(1), 108-111.

OECD. (2020). OECD Data: Fertility rates 1970-2015. Retrieved December 30, 2020 from https://data.oecd.org/pop/fertility-rates. htm

Okun, B. S. (2013). Fertility and marriage behavior in Israel: diversity, change, and stability. Demographic Research, 28, 457-504. 
Payne, P., Levine, P. A., \& Crane-Godreau, M. A. (2015). Somatic experiencing: using interoception and proprioception as core elements of trauma therapy. Frontiers in Psychology, 6, 93.

Pazzagli, C., Delvecchio, E., Raspa, V., Mazzeschi, C., \& Luyten, P. (2018). The parental reflective functioning questionnaire in mothers and fathers of school-aged children. Journal of Child and Family Studies, 27(1), 80-90.

Pietrzak, R. H., Goldstein, R. B., Southwick, S. M., \& Grant, B. F. (2011). Prevalence and Axis I comorbidity of full and partial posttraumatic stress disorder in the United States: results from Wave 2 of the National Epidemiologic Survey on Alcohol and Related Conditions. Journal of Anxiety Disorders, 25(3), 456-465.

Rostad, W. L., \& Whitaker, D. J. (2016). The association between reflective functioning and parent-child relationship quality. Journal of Child and Family Studies, 25(7), 2164-2177.

Rutherford, H. J., Byrne, S. P., Crowley, M. J., Bornstein, J., Bridgett, D. J., \& Mayes, L. C. (2018). Executive functioning predicts reflective functioning in mothers. Journal of Child and Family Studies, 27(3), 944-952.

Rutherford, H. J., Goldberg, B., Luyten, P., Bridgett, D. J., \& Mayes, L. C. (2013). Parental reflective functioning is associated with tolerance of infant distress but not general distress: evidence for a specific relationship using a simulated baby paradigm. Infant Behavior and Development, 36(4), 635-641.

Rutherford, H. J., Maupin, A. N., Landi, N., Potenza, M. N., \& Mayes, L. C. (2017). Parental reflective functioning and the neural correlates of processing infant affective cues. Social Neuroscience, 12(5), 519-529.

Saunders, J. B., Aasland, O. G., Babor, T. F., De la Fuente, J. R., \& Grant, M. (1993). Development of the alcohol use disorders identification test (AUDIT): WHO collaborative project on early detection of persons with harmful alcohol consumption-II. Addiction, 88(6), 791-804.

Schechter, D. S., Coots, T., Zeanah, C. H., Davies, M., Coates, S. W., Trabka, K. A., Marshall, R. D., Liebowitz, M. R., \& Myers, M. M. (2005). Maternal mental representations of the child in an inner-city clinical sample: Violence-related posttraumatic stress and reflective functioning. Attachment \& Human Development, 7(3), 313-331.

Seal, K. H., Cohen, G., Waldrop, A., Cohen, B. E., Maguen, S., \& Ren, L. (2011). Substance use disorders in Iraq and Afghanistan veterans in VA healthcare, 2001-2010: Implications for screening, diagnosis and treatment. Drug and Alcohol Dependence, 116 (1-3), 93-101.

Sherman, M. D., Gress Smith, J. L., Straits-Troster, K., Larsen, J. L., \& Gewirtz, A. (2016). Veterans' perceptions of the impact of PTSD on their parenting and children. Psychological Services, 13 (4), 401.

Skipper, L. D., Forsten, R. D., Kim, E. H., Wilk, J. D., \& Hoge, C. W. (2014). Relationship of combat experiences and alcohol misuse among US special operations soldiers. Military Medicine, 179(3), 301-308.
Stover, C. S., \& Kiselica, A. (2014). An initial examination of the association of reflective functioning to parenting of fathers. Infant Mental Health Journal, 35(5), 452-461.

Suardi, F., Moser, D. A., Sancho Rossignol, A., Manini, A., Vital, M., Merminod, G., Kreis, A., Ansermet, F., Rusconi Serpa, S., \& Schechter, D. S. (2020). Maternal reflective functioning, interpersonal violence-related posttraumatic stress disorder, and risk for psychopathology in early childhood. Attachment \& Human Development, 22(2), 225-245.

Taraban, L., \& Shaw, D. S. (2018). Parenting in context: revisiting Belsky's classic process of parenting model in early childhood. Developmental Review, 48, 55-81.

Teti, D. M., \& Candelaria, M. A. (2002). Parenting competence. In Bornstein M. H. (Ed.) Handbook of Parenting. Volume 4: Social Conditions and Applied Parenting.

Tomás, M. T. C., Costa, J. A. G., Motos-Sellés, P., Beitia, M. D. S., \& Mahía, F. C. (2017). The utility of the alcohol use disorders identification test (AUDIT) for the analysis of binge drinking in university students. Psicothema, 29(2), 229-235.

Wagner, T. H., Harris, K. M., Federman, B., Dai, L., Luna, Y., \& Humphreys, K. (2007). Prevalence of substance use disorders among veterans and comparable nonveterans from the National Survey on Drug Use and Health. Psychological Services, 4(3), 149.

Weathers, F. W., Litz, B. T., Keane, T. M., Palmieri, P. A., Marx, B. P., \& Schnurr, P. P. (2013). The ptsd checklist for dsm-5 (pcl-5). Scale available from the National Center for PTSD at www.ptsd. va.gov, 10.

Wortmann, J. H., Jordan, A. H., Weathers, F. W., Resick, P. A., Dondanville, K. A., Hall-Clark, B., Foa, E. B., YoungMcCaughan, S., Yarvis, J. S., \& Hembree, E. A. (2016). Psychometric analysis of the PTSD Checklist-5 (PCL-5) among treatment-seeking military service members. Psychological Assessment, 28(11), 1392.

Wright, K. M., Foran, H. M., Wood, M. D., Eckford, R. D., \& McGurk, D. (2012). Alcohol problems, aggression, and other externalizing behaviors after return from deployment: understanding the role of combat exposure, internalizing symptoms, and social environment. Journal of Clinical Psychology, 68(7), 782-800.

Xiong, J., Lipsitz, O., Nasri, F., Lui, L. M., Gill, H., Phan, L., Chen-Li, D., Iacobucci, M., Ho, R., \& Majeed, A. (2020). Impact of COVID-19 pandemic on mental health in the general population: a systematic review. Journal of Affective Disorder. https://doi. org/10.1016/j.jad.2020.08.001.

Yehuda, R., Hoge, C. W., McFarlane, A. C., Vermetten, E., Lanius, R. A., Nievergelt, C. M., Hobfoll, S. E., Koenen, K. C., Neylan, T. C., \& Hyman, S. E. (2015). Post-traumatic stress disorder. Nature Reviews Disease Primers, 1(1), 1-22.

Zerach, G., \& Levi-Belz, Y. (2018). Moral injury process and its psychological consequences among Israeli combat veterans. Journal of Clinical Psychology, 74(9), 1526-1544. 